\title{
Surgical Treatment Options for Patients with Large and Giant Postoperative Reducible Medial Abdominal Hernias
}

\author{
Evgeniy F. Cherednikov, $\mathrm{PhD}, \mathrm{ScD}^{1}$; Galina V. Polubkova, $\mathrm{PhD}^{1}$; Stanislav A. \\ Skorobogatov, $\mathrm{MD}^{2}$; Ludmila E. Mehantjeva, $\mathrm{PhD}, \mathrm{ScD}^{1}$; Evgeniy S. Ovsyannikov, $\mathrm{PhD}^{1^{*}}$ \\ ${ }^{1}$ Voronezh State Medical University \\ ${ }^{2}$ Voronezh City Clinical Emergency Hospital №10 \\ Voronezh, the Russian Federation
}

\begin{abstract}
The aim of the study was to assess surgical treatment outcomes of patients with large and giant postoperative reducible medial abdominal hernias based on the developed algorithm for selecting the optimal method of hernioplasty.

Materials and Methods: The study included 31 patients with large and giant postoperative reducible medial abdominal hernias. Before and after the operation, all patients underwent an electromyographic assessment of the functional state of the anterior abdominal wall muscles with determination of the muscle fatigue index (FI). FI $\leq 2.4$ indicated the preserved muscle function. In addition, on patients of the experimental group we performed a simulation of the postoperative situation with determination of the intra-abdominal pressure (IAP) level by measuring blood oxygen saturation $\left(\mathrm{SpO}_{2}\right)$. A specific method of plastic surgery was chosen based on the obtained parameters of the increase in FI and IAP levels (a decrease in $\mathrm{SpO}_{2}$ ). The adequacy of the surgical treatment option was supported by monitoring the $\mathrm{SpO}_{2}$ level in the early postoperative period (Days 1, 3, and 7). Absence of a significant increase in IAP and adequate reduction in the $\mathrm{SpO}_{2}$ level indicated the proper choice of plastic technique.

Results: In the patients of the experimental group, who were operated on with reconstructive plastic surgery, the average parameters of FI of the abdominal wall muscles were within 1.89|1.68|2.0 $(P<0.05)$, which is evidence for the preservation of muscle function and an adequate choice of the surgical treatment option. In patients of the comparison group, who had been operated on with corrective methods of surgical treatment, the parameters of FI were within $2.79|2.52| 3.16(P<0.01)$, which is evidence for the loss of muscle function of the anterior abdominal wall.

Conclusion: The proposed algorithm for choosing a method of hernioplasty based on the width of the hernia orifice and preoperative assessment of additional parameters, such as the functional state of the anterior abdominal wall muscles and the level of $\mathrm{SpO}_{2}$, in simulation of the postoperative situation allowed for a differentiated, functionally oriented approach toward the choice of surgical treatment options in patients with large and giant postoperative reducible medial abdominal hernias. (International Journal of Biomedicine. 2019;9(3):237-241.)
\end{abstract}

Key Words: postoperative reducible medial abdominal hernias $\bullet$ hernioplasty $\bullet$ blood oxygen saturation $\bullet$ electromyography

\section{Introduction}

The problem of surgical treatment of the anterior abdominal wall hernias remains one of the most challenging today. ${ }^{(1-4)}$ Currently, surgical interventions for postoperative medial abdominal hernias are considered to be among the

*Corresponding author: Evgeniy S. Ovsyannikov, PhD. Department of Faculty Therapy, Voronezh State Medical University named after N.N. Burdenko. Voronezh, Russia. E-mail: ovses@ yandex.ru most commonly performed procedures. ${ }^{(1,5,6)}$ It is known that operations for large and giant postoperative hernias are complex and rather dangerous, accompanied by a large number of postoperative complications (15-33\%), a significant number of hernia relapses (18-44\%) and high mortality rates $(2-5.8 \%) .{ }^{(7)}$ The recurrence rate in elderly patients reaches $45 \%$ or more. ${ }^{(8,9)}$

Despite the large number of surgical treatment options for postoperative medial hernias, there is no unified approach to the choice of plastic surgery options nowadays. Determination of the optimal variant of a plastic option for a hernia defect is 
possible only after a detailed assessment of a patient's general condition. $^{(10)}$ In the treatment of postoperative medial hernias, the "tension-free" technique of prosthetic abdominal wall hernia repair has become widely spread..$^{(11,12)}$

In large and giant postoperative hernias, a sharp increase in intra-abdominal pressure (IAP) resulting from immersing the hernia sac into the abdominal cavity and bringing the borders of the hernia orifice closer together appears to be a dangerous complication of hernioplasty in the postoperative period. Therefore, currently, the treatment of these patients must necessarily be accompanied by control of the magnitude and dynamics of IAP at various stages of treatment. ${ }^{(13)}$ In clinical practice, the method of indirect IAP assessment by the bladder pressure measurement using a transurethral catheter ${ }^{(7)}$ has become most prevalent. However, this method has several disadvantages: it is invasive, poorly tolerated by patients, and frequently results in complications. We applied a non-invasive, simple and effective method for an indirect IAP assessment - measurement of the $\mathrm{SpO}_{2}$ level. ${ }^{(15,16)}$ In addition, restoring function of the abdominal wall muscle after hernioplasty is of great significance in the treatment of patients with large hernias..$^{(5,6,14,15)}$

\section{Materials and Methods}

The study included 31 patients with large and giant postoperative reducible medial abdominal hernias. The study was carried out in compliance with Ethical Principles for Medical Research Involving Human Subjects, Adopted by the 18th WMA General Assembly, Helsinki, Finland, June 1964, and amended by the 52nd WMA General Assembly, Edinburgh, Scotland, October 2000. The study was approved by the Ethics Committee of Voronezh State Medical University named after N.N. Burdenko. All patients gave their written informed consent.

The patient cohort consisted of $11(35.5 \%)$ male patients and 20(64.5\%) female patients. The patients were distributed based on their age, in accordance with WHO classification, 2012. The mean age was $56.19 \pm 2.88$ years. Analysis of the age composition demonstrated that the majority of patients $(61.3 \%)$ were of active working age (from 25 to 60 years). This indicates the social significance of the problem discussed.

According to the generally accepted classification of the European Hernia Society (EHS), all patients had an $\mathrm{M}_{2-4} \mathrm{~W}_{3} \mathrm{R}_{0}$ hernia. Depending on their location on the abdominal wall, all hernia defects were medial hernias located in the epigastric, umbilical and infraumbilical regions. In all patients, the width of the hernia was large $(\geq 10 \mathrm{~cm})$. All hernias were nonrecurrent $\left(\mathrm{R}_{0}\right)$.

As for the structure of comorbidities, hypertension $(45.2 \%)$ and coronary heart disease $(38.7 \%)$ prevailed in patients included in the study. Obesity (29\%), diabetes mellitus (25.8\%) and gastrointestinal diseases (16.1\%) were also quite common. The study did not include patients with diseases of the broncho-pulmonary system in order to obtain more accurate results when determining IAP for $\mathrm{SpO}_{2}$.

According to the objectives of the study, all patients were randomly divided into two groups: the experimental group $(n=17)$ and the comparison group $(n=14)$. All patients in both groups had comparable size and location of the hernia orifice, comorbidities and age.

In the experimental group, the choice of plastic treatment option was based not only on the width of the hernia orifice (the standard approach), but also on consideration of other parameters: 1) the functional state of the abdominal wall muscles before the operation and 2) probable level of the increase in IAP with simultaneous elimination of the hernia.

To assess the functional state of the anterior abdominal wall muscles, electromyography was performed before the operation and 3 months after it, using the Neurosoft electromyograph Neuro-MEP. The study was based on the determination of the muscle fatigue index (FI). If the muscle $\mathrm{FI} \leq 2.4$, the muscle function is preserved; if the muscle $\mathrm{FI}>2.4$, the function is lost. ${ }^{(6)}$

To determine the probable level of IAP increase, we simulated the forthcoming postoperative situation by immersing the hernial protrusion into the abdominal cavity using a bandage and simultaneously bringing the edges of the hernia orifice close together with a bandage to control the patient's abdomen. To assess the degree of IAP increase, $\mathrm{SpO}_{2}$ was recorded twice: before and after simulation. A corresponding decrease in $\mathrm{SpO}_{2}$ resulting from the IAP increase was observed under simulation and recorded..$^{(5,15)}$ Blood oxygen saturation was measured using pulse oximeters OP-31.1 "Triton T-31" (Russia) and Bitmos Sat 816.

Having analyzed the functional state of the abdominal muscle and the $\mathrm{SpO}_{2}$ parameters obtained under simulation of the forthcoming postoperative situation, we developed an algorithm for a more accurate selection of the plastic surgery option. In addition, when choosing a method of surgical treatment, we always sought to restore the normal anatomy of the anterior abdominal wall and preserve the muscle functionthus, providing a functionally oriented approach. ${ }^{(17)}$

With large and giant hernias, this became possible using the 2-stage hardware technique developed by E.N. Lyubykh, 2013. ${ }^{(6)}$ This hardware method allows avoiding negative side effects of both tension (a sharp IAP increase and strong tissue tension in the plastics zone) and "tension-free" plastics (use of large-sized meshes and preservation of the pathological anatomy of the anterior abdominal wall).

In the experimental group, we applied the following surgical treatment options for eliminating the hernia:

1. The 2-stage hardware technique. ${ }^{(6)}$ At the first stage, devices were applied to patients to gradually converge of the edges of the hernia orifice in order to prevent a sharp IAP increase. In this case, the hernia was immersed in the abdominal cavity with a bandage. This technique was applied daily, by $1 \mathrm{~cm}$ per day for 12-14 days until the edges of the hernia orifice fully converged. This allowed patients to gradually adapt to the IAP increase, avoiding the development of acute respiratory failure. At the second stage of treatment, hernia repair was performed-plastic surgery using local tissue flaps with restoration of the normal anatomy of the abdominal wall and reinforcement of prosthesis with a smallsized mesh. ${ }^{(17) .}$ 
2. Posterior separation plastics (with retromuscular placement of the endoprosthesis). This method allowed restoring normal white-line anatomy, but was more traumatic due to tissue separation and partial violation of innervation; moreover, it required a larger-sized mesh prosthesis. ${ }^{(12)}$

3. Endoprosthetics of the anterior abdominal wall (with intramuscular placement of a mesh prosthesis) in the form of a "patch." The method retained the existing pathological anatomy of the anterior abdominal wall and required a largesized mesh prosthesis. ${ }^{(11,12)}$

In the experimental group, the IAP level was assessed using $\mathrm{SpO}_{2}$ to validate the correctness of the plastic surgery option on Days 1, 2 and 7 after the operation.

To assess the obtained functional results of treatment, the state of the abdominal wall muscle was determined using electromyography in the late postoperative period (3 months after the operation).

In the comparison group, we applied a standard approach when choosing a plastic surgery option, focusing mainly on the width of the hernia orifice. Considering the large width of the hernia orifice $\left(\mathrm{W}_{3}\right)$, corrective operations (herniotomy and abdominal wall prosthetics with a mesh prosthesis) were performed for all patients in order to exclude a sharp IAP increase (compartment syndrome) in the early postoperative period. In the comparison group, $\mathrm{SpO}_{2}$ was determined one time before the operation and 3 times after the operation (on Days 1, 2, and 7). In this group, simulation of the postoperative situation was not performed. To assess the functional state of the abdominal wall muscles, electromyography was performed twice: before and 3 months after the operation.

The statistical analysis was performed using the statistical software Microsoft Excel. The results are presented as median (Me), upper (UQ) and lower (LQ) quartiles. The Mann-Whitney (U Test) was used to compare the differences between the two independent groups. The Wilcoxon criterion was used to compare the differences between the paired samples. Group comparisons with respect to categorical variables are performed using chi-square tests or, alternatively, Fisher's exact test when expected cell counts were less than 5. A probability value of $P<0.05$ was considered statistically significant.

\section{Results}

In the experimental group, $\mathrm{SpO}_{2}$ in all patients was within the normal range before the operation: $98|97.5| 98.2 \%$ $(\mathrm{Me}|\mathrm{UQ}| \mathrm{LQ})(P<0.05)$. When simulating the postoperative situation, there was registered a decrease in $\mathrm{SpO}_{2}(87.4|87| 88 \%)$ $(P<0.01)$, which is evidence for a significant IAP increase. ${ }^{(16)}$ Therefore, in patients of this group, simultaneous plastic surgery with restoration of the normal anatomy of the abdominal wall using conventional tension plastics could not be performed due to the high risk of developing the compartment syndrome. In such situations, it is possible to perform only the operations that allow avoiding a sharp IAP increase in the early postoperative period. These operations involve the 2-stage hardware technique, posterior separation plastics, and endoprosthetics of the abdominal wall with a mesh prosthesis.
The results of electromyography of the abdominal muscles gave an opportunity for a more accurate choice of the plastic surgery option. If electromyography findings demonstrated that the muscle function was preserved, then plastic surgery options with the normal anatomy restoration were indicated to preserve and further improve the abdominal muscle function. If electromyography findings demonstrated that the muscle function was already lost, then the restoration of normal anatomy would not result in the restoration of a function, and a simpler and less traumatic plastic surgery option was indicated, i.e. mesh prosthetics.

Of the patients in the experimental group, 10(58.8\%) demonstrated preserved muscle function: according to electromyography findings, their FI values were within $2.28|2.12| 2.35(P<0.01)$. This allowed applying reconstructive methods of plastics with the restoration of normal anatomy in these patients.

The 2-stage hardware technique was performed in 7 of these 10 patients; they revealed a significant decrease in blood saturation during simulation of the postoperative situation $(87.2|87| 87.35 \%)(P<0.05)$ with the preserved function of the anterior wall muscles (muscle FI values were within 2.29|2.2|2.33) $(P<0.05)$.

Posterior separation plastic was performed in 3 out of 10 patients; they experienced a less significant decrease in $\mathrm{SpO}_{2}$ during stimulation of the postoperative situation $(89.5|89.25| 89.85 \% ; P=0.05)$ and normal parameters of the functional state of the abdominal muscles(muscle FI values were within $2.12|2.04| 2.24 ; P<0.05)$.

The remaining $7(41.2 \%)$ patients of the experimental group had almost lost muscle function by the time of the operation. (muscle FI values were within 2.58|2.52|2.73) $(P<0.05)$. In addition, they experienced a significant decrease in $\mathrm{SpO}_{2}$ during simulation of the postoperative situation: $87.5|87.1| 87.75 \%(P<0.05)$; therefore, a mesh prosthetics of the abdominal wall was performed on these patients (corrective surgery).

No sharp rise in IAP was registered after performing the hernia repair and hernioplasty using the 2-stage hardware technique since the patients were prepared by the first stage of the operation. On Day 1 , their $\mathrm{SpO}_{2}$ constituted $92.2|92.05| 92.35 \%(P<0.05)$; this required oxygen inhalation. On Day 2, $\mathrm{SpO}_{2}$ constituted $93.7|93.55| 94 \%(P<0.05)$; on Day 7 $\mathrm{SpO}_{2}$ constituted $96.5|96.25| 96.8 \%(P<0.05)$; these values were close to the parameters before the operation- $-98.2|98| 98.35 \%$ $(P<0.05)$.

After posterior separation plastics, $\mathrm{SpO}_{2}$ constituted 91.7|91.6|91.85\% $(P=0.05)$ on Day 1 after the operation, which required oxygen inhalation; on Day 2 after the operation the parameters improved: $\mathrm{SpO}_{2}$ values were within $93|92.9| 93.2 \%$ $(P=0.05)$. An increase in $\mathrm{SpO}_{2}$ was recorded on Day 7 and constituted of $96|95.85| 96.1 \%(P=0.05)$; these values were also close to the preoperative parameters-98.2|97.85|98.25\% $(P<0.05)$.

After mesh prosthetics, $\mathrm{SpO}_{2}$ constituted $92|91.85| 92.15 \%$ $(P<0.05)$ on Day 1, which required oxygen inhalation; on Day 2, $\mathrm{SpO}_{2}$ was within $93.5|93.35| 93.65 \%(P<0.05)$. On Day 7, $\mathrm{SpO}_{2}$ was recorded within $95.8|95.5| 96 \%(P<0.05)$; these values were 
close to the parameters before the operation $-97.6|97.4| 97.75 \%$ $(P<0.05)$.

The absence of a sharp increase in IAP in the early postoperative period under various methods of plastics in patients of the experimental group indicates the adequacy of the plastic surgery option.

In the late postoperative period (after 3 months), all patients of the experimental group underwent an electromyographic assessment of the functional state of the anterior abdominal wall muscles. After reconstructive surgeries, the muscle function was preserved. After the 2-stage hardware technique, the FI parameters of the abdominal muscles were 1.78|1.64|1.97 $(P<0.05)$; after a posterior separation plastic, the abdominal muscle FI parameters were $1.92|1.89| 2.02 \quad(P<0.05)$, a fact that supports the adequacy of the surgical treatment option. Muscle function was lost (muscle FI was $-2.73|2.69| 3.04)(P<0.05)$ in patients of the experimental group, who received corrective treatment.

In the comparison group, $\mathrm{SpO}_{2}$ was within the normal range of $97.85|97.5| 98.15 \%(P<0.05)$ in all patients before the operation. After corrective plastics, $\mathrm{SpO}_{2}$ constituted $89.65|89.35| 90.2 \% \quad(P<0.05)$ on Day 1; on Day 2, $\mathrm{SpO}_{2}$ parameters were $91.75|91.4| 92 \%(P<0.05)$, which required oxygen inhalation. On Day $7, \mathrm{SpO}_{2}$ parameters were 95.9|95.35|96.2\% $(P<0.05)$; these parameters were close to the preoperative values.

In the comparison group, muscle function had already been lost prior to the operation in $8(57.1 \%)$ patients: according to the electromyography findings, the abdominal muscle FI values were within 2.92|2.82|3.0 $(P<0.05)$. The remaining $6(42.9 \%)$ patients of the comparison group demonstrated preserved muscle function: according to the electromyography findings, FI values were within $2.32|2.29| 2.34(P<0.05)$. All patients were given corrective treatment, and in 3 months, the muscle function was evaluated by electromyography. Eight patients (57.1\%) of the comparison group, who demonstrated lost muscle function before the operation, did not have their muscle function restored within 3 months after the operation; moreover, their FI values slightly deteriorated and constituted 3.15|2.97|3.22 $(P<0.05)$. In $6(42.9 \%)$ patients of the comparison group, with preserved muscle function, muscle function moderately deteriorated within 3 months after surgery: the FI values were $2.48|2.45| 2.56(P<0.05)$.Concurrently, application of a dynamic load detected a lower level of the rectus abdominus muscle biopotentials in all patients of the comparison group; this fact supports their failure to completely perform their functions.

\section{Conclusion}

The proposed algorithm for choosing a method of hernioplasty based on the width of the hernia orifice and preoperative assessment of additional parameters, such as the functional state of the anterior abdominal wall muscles and the level of $\mathrm{SpO}_{2}$, in simulation of the postoperative situation allowed for a differentiated, functionally oriented approach toward the choice of surgical treatment options in patients with large and giant postoperative reducible medial abdominal hernias.

\section{Competing Interests} interests.

The authors declare that they have no competing

\section{References}

1. Gogia BSh, Alyautdinov RR, Kopyltsov AA, Tokareva TV. New technologies in the treatment postoperative abdominal wall hernias. Vysokotekhnologicheskaya Meditsina. 2016;3. [Article in Russian].

2. Polubkova GV, Cherednikov EF, Kashurnikova MA. Physical rehabilitation of patients after laparoscopic hernioplasty for inguinal hernias. Proceedings of the V AllRussian Distant Scientific and Practical Conference with international participation "Biomedical and pedagogical bases of adaptation, sports activities and a healthy lifestyle". Voronezh; 2016:288-291. [Article in Russian].

3. Polubkova GV, Cherednikov EF, Kashurnikova MA, Shlykov OA, Usov YV, Tkachev IE, et al. Evaluation of the results of laparoscopic transabdominal preperitoneal plasty in the treatment of patients with inguinal hernias. Nauchnomeditsinskiy vestnik Tsentralnogo Chernozemya. Voronezh. 2016;(65):10-13. [Article in Russian].

4. Strygin OV, Polubkova GV, Mikhailova GN, Cherednikov EF, Gulyaev AA., Krasikov AI, et al. Experience of application of topographically and anatomically based, functionallyoriented methods of plasty of inguinal hernias. Nauchnomeditsinskiy vestnik Tsentralnogo Chernozemya. Voronezh. 2016;(65):14-19. [Article in Russian].

5. Cherednikov EF, Polubkova GV, Glukhov AA, Skorobogatov SA. Selecting the Way to Surgical Treatment of Patients with Postoperative the Median Rectifiable Hernia Based Noninvasive Evaluation of Intra-abdominal Pressure. Journal of Experimental and Clinical Surgery. 2017;10 (2):103-110. [Article in Russian].

6. Lyubykh EN, Komkova EA, Slepokurova TA. (2013). The method of selecting the type of surgical treatment of patients with hernial defects of the anterior abdominal wall. Russian Federation Patent \# 2491887 (25).2013. [In Russian].

7. Gelfand BR, Protsenko DN, Podachin PV. Abdominal hypertension syndrome: the state of the problem. Medical alphabet. Emergency Medicine]. 2010; 3: 34-42. [Article in Russian].

8. Berger D, Lux A. [Operative therapy of secondary ventral hernia: technical principles]. Chirurg. 2013;84(11):10011012. doi: 10.1007/s00104-011-2245-y. [Article in German].

9. Hanna EM, Byrd JF, Moskowitz M, Mann JW, Stockamp KT, Patel GN, et al. Outcomes of a prospective multi-center trial of a second-generation composite mesh for open ventral hernia repair. Hernia. 2014 Feb;18(1):81-9. doi: 10.1007/ s10029-013-1078-7.

10. Shestakov AL, Inakov AG, Ckhovrebov AT. Estimation of the effectiveness of hernioplasty in patients with hernias of the anterior abdominal wall using the SF-36. Bulletin of Pirogov National Medical \& Surgical Center. 2017; 12 (3): 50-53. [Article in Russian].

11. Belokonev VI, Vavilov AV, Gulyaev MG, Grachev DB. Tactics of treatment of patients with recurrent postoperative 
incisional hernias. Tavricheskiy mediko-biologicheskiy vestnik. 2018;21(2):17-23. [Article in Russian].

12. Zakurdaev EI, Chernyh AV, Cherednikov EF, Zakurdaeva MP. Topographic-Anatomic Justification of Various Methods Reducing Tissue Tension at Prosthetic Incisional Hernia Repair by «Sublay». Journal of Experimental and Clinical Surgery. 2017;10(1):26-31. [Article in Russian].

13. Malbrain MLNG, Jones F. Intra-abdominal pressure measurement techniques. In: Ivatury RR, Cheatham ML, Malbrain MLNG, Sugrue M, editors. Abdominal Compartment Syndrome. Landes Biomedical, Georgetown, 2006.

14. Timoshin AD, Shestakov AL, Zagorulko OI., Inakov AG. Abdominal wall muscles function estimation after hernioplasty. Bulletin of Siberian Medicine. 2007;3:62.
[Article in Russian].

15. Cherednikov EF, Polubkova GV, Skorobogatov SA, Novikova EI. Results the application to non-invasive evaluation of intra-abdominal pressure in the surgical treatment of patients with postoperative the median free hernia. System analysis and management in biomedical systems]. 2017; 16(2):328-334. [Article in Russian].

16. Skorobogatov S.A., Lyubykh E.N., Baskakov E.I. A method for assessing intra-abdominal pressure in patients with abdominal hernias. Russian Federation Patent \#2575336 (5). 2016. [In Russian].

17. Lyubyh EN, Polubkova GV. On some problematic issues of herniology and ways to solve them. Gerniologiya. 2009;2(22):14-17. [Article in Russian]. 